University of Nebraska - Lincoln

DigitalCommons@University of Nebraska - Lincoln

Robert Streubel Papers

Research Papers in Physics and Astronomy

2-1-2021

\title{
Chiral Spin Textures in Amorphous Iron-Germanium Thick Films
}

\author{
Robert Streubel \\ University of Nebraska-Lincoln, streubel@unl.edu \\ D. Simca Bouma \\ Lawrence Berkeley National Laboratory \\ Frank Bruni \\ University of California, Berkeley \\ Xiaoqian Chen \\ Lawrence Berkeley National Laboratory \\ Peter Ercius \\ Lawrence Berkeley National Laboratory
}

See next page for additional authors

Follow this and additional works at: https://digitalcommons.unl.edu/physicsstreubel

Part of the Atomic, Molecular and Optical Physics Commons, Condensed Matter Physics Commons, and the Other Physics Commons

Streubel, Robert; Bouma, D. Simca; Bruni, Frank; Chen, Xiaoqian; Ercius, Peter; Ciston, Jim; N'Diaye, Alpha T.; Roy, Sujoy; Kevan, Steve D.; Fischer, Peter; and Hellman, Frances, "Chiral Spin Textures in Amorphous Iron-Germanium Thick Films" (2021). Robert Streubel Papers. 27.

https://digitalcommons.unl.edu/physicsstreubel/27

This Article is brought to you for free and open access by the Research Papers in Physics and Astronomy at DigitalCommons@University of Nebraska - Lincoln. It has been accepted for inclusion in Robert Streubel Papers by an authorized administrator of DigitalCommons@University of Nebraska - Lincoln. 


\section{Authors}

Robert Streubel, D. Simca Bouma, Frank Bruni, Xiaoqian Chen, Peter Ercius, Jim Ciston, Alpha T. N'Diaye, Sujoy Roy, Steve D. Kevan, Peter Fischer, and Frances Hellman 


\title{
Chiral Spin Textures in Amorphous Iron-Germanium Thick Films
}

\author{
Robert Streubel, D. Simca Bouma, Frank Bruni, \\ Xiaoqian Chen, Peter Ercius, Jim Ciston, \\ Alpha T. N’Diaye, Sujoy Roy, Steve D. Kevan, \\ Peter Fischer, and Frances Hellman
}

Prof. R. Streubel

Department of Physics and Astronomy, and Nebraska Center for

Materials and Nanoscience, University of Nebraska-Lincoln, Lincoln, NE 68588, USA

E-mail: streubel@unl.edu (Corresponding author)

Prof. R. Streubel, Dr. D.S. Bouma, Dr. X. Chen, Dr. P. Fischer

Materials Sciences Division, Lawrence Berkeley National Laboratory,

Berkeley, CA 94720, USA

Dr. D.S. Bouma, F. Bruni, Prof. F. Hellman

Department of Physics, University of California Berkeley, Berkeley, CA 94720, USA

Dr. P. Ercius, Dr. J. Ciston

National Center for Electron Microscopy, Molecular Foundry, Lawrence Berkeley National Laboratory, Berkeley, CA 94720, USA

Dr. X. Chen, Dr. A.T. N’Diaye, Dr. S. Roy, Dr. S.D. Kevan

Advanced Light Source, Lawrence Berkeley National Laboratory,

Berkeley, CA 94720, USA

Published in Advanced Materials 2021, 33, 2004830

DOI: $10.1002 / a d m a .202004830$

Copyright (C) 2021 Wiley-VCH GmbH. Used by permission.

Submitted July 15, 2020; revised December 2, 2020; published January 12, 2021. 


\begin{abstract}
Topological solitary fields, such as magnetic and polar skyrmions, are envisioned to revolutionize microelectronics. These configurations have been stabilized in solid-state materials with a global inversion symmetry breaking, which translates in magnetic materials into a vector spin exchange known as the Dzyaloshinskii-Moriya interaction (DMI), as well as spin chirality selection and isotropic solitons. This work reports experimental evidence of 3D chiral spin textures, such as helical spins and skyrmions with different chirality and topological charge, stabilized in amorphous Fe-Ge thick films. These results demonstrate that structurally and chemically disordered materials with a random DMI can resemble inversion symmetry broken systems with similar magnetic properties, moments, and states. Disordered systems are distinguished from systems with global inversion symmetry breaking by their degenerate spin chirality that allows for forming isotropic and anisotropic topological spin textures at remanence, while offering greater flexibility in materials synthesis, voltage, and strain manipulation.
\end{abstract}

Keywords: amorphous materials, coherent X-ray scattering, local inversion symmetry breaking, Lorentz microscopy, topological magnetic states, X-ray spectro-microscopy

The discovery of unprecedented physical properties and application potential of topologically protected non-collinear states in condensed matter has greatly influenced the direction of basic sciences of magnetic and ferroelectric materials. Magnetic ${ }^{[1-9]}$ and polar ${ }^{[10,11]}$ skyrmions have been examined in a large variety of materials systems and manipulation by external stimuli, including current, voltage, and strain. The latter is both fundamentally intriguing and relevant to novel information storage and processing units, such as the racetrack memory ${ }^{[12,13]}$ which harnesses electromagnetism in solids ${ }^{[14,15]}$ to link topological properties to electronic transport phenomena. To date, the emergence of topological vector fields has almost exclusively been associated with a global symmetry breaking, following the original works by Skyrme ${ }^{[16]}$ and Faddeev, ${ }^{[17]}$ that causes in magnetic systems with large spin-orbit coupling ${ }^{[18,19]} \mathrm{a}$ vector spin exchange interaction, known as the Dzyaloshinskii-Moriya interaction (DMI). ${ }^{[20,21]}$ This approach imposes two fundamental constraints: 1) magnetic materials must break inversion symmetry, either by virtue of their crystal structure, ${ }^{[1]}$ as in B20 FeGe, ${ }^{[22-25]}$ or through the presence of interfaces, ${ }^{[26,27]}$ as in thin multilayer stacks; ${ }^{[6-8,28-30]}$ 2) the emergent chirality selection prevents the stabilization of higherorder, anisotropic topological states that continuously vary spin chirality and spin direction. Exploring these anisotropic solitary spin textures, 
such as skyrmions with large topological charge $N$ and 3D topological knots referred to as hopfions, ${ }^{[31,32]}$ in condensed matter is the next step in this endeavor, simultaneously promising technological impacts.

Systems with a locally varying $\mathrm{DMI}^{[33,34]}$ or a spontaneous symmetry breaking with respect to spin chirality bear great potential to stabilize twisted and anisotropic magnetic solitons beyond biskyrmions. [35-37] The spontaneous symmetry breaking can be accomplished in two ways: 1) realizing a higher-order exchange interaction with alternating sign of Heisenberg exchange between nearest and next nearest neighbors as proposed in theoretical works; ${ }^{[31,38,39]}$ or as shown here, 2) decreasing the Heisenberg exchange and inducing a random DMI between adjacent atoms in structurally and chemically disordered systems. In this context, randomness refers to both orientation and strength of the local DMI, which can be pictured as an inhomogeneous vector spin exchange governed by local structural and chemical order. We have recently shown an onset of magnetic order in amorphous $\mathrm{Fe}_{x} \mathrm{Ge}_{1-x}$ films at $x \approx 0.4(2 \mathrm{~K})$, giving rise to a large intrinsic anomalous Hall effect and exhibiting non-saturating S-shaped hysteresis loops which suggest the presence of non-collinear spin textures. ${ }^{[40]}$ The challenge is to tailor the exchange interactions to favor long-range ordering of chiral spin textures over spin frustration prevailing in traditional spin glass materials. Magnetic ordering of chiral spins was previously demonstrated in geometrically frustrated XY dipolar spin systems with structural disorder. ${ }^{[41-43]}$

Here, we report experimental evidence of helical structures and topological magnetization vector fields in structurally and chemically disordered amorphous 80 nm-thick $\mathrm{Fe}_{x} \mathrm{Ge}_{1-x}$ films $(0.52 \lesssim x \lesssim 0.68)$. In contrast to previous works on multilayer stacks or crystalline materials, the present sample system does not possess a well-defined symmetry or inversion symmetry breaking but takes advantage of local inversion symmetry breaking and DMI. Harnessing Lorentz microscopy with exit wave reconstruction, we observe both isotropic Bloch skyrmions $(N=1)$, previously found in B20 single-crystals, and anisotropic solitons, such as antiskyrmions $(N=-1)$ and $N=2$ skyrmions. We employ magnetometry and X-ray magnetic circular dichroism (XMCD) spectroscopy to quantify the magnetic anisotropy and magnetic moments that suggest a short-range order similar to B20 FeGe single-crystals despite lacking a global broken chiral symmetry. The emergent magnetic 
order of the chiral spin textures is accompanied by a reduced orbitalto-spin moment ratio, underling the importance of disordered electron orbitals and random DMI. Joint studies with Lorentz microscopy and resonant coherent X-ray scattering, offering in-plane and out-ofplane sensitivity to the magnetization, respectively, identify a phase transition in $x=0.52$ samples from a high-temperature helical phase to a low-temperature state that lacks, with the exception of localized spin excitations, both long-range and short-range magnetic order. The thermal spin fluctuations below the phase transition show a persistent switching of $N=2$ skyrmions between two discrete states that share the same symmetry axis. Different orientations of the symmetry axis and locally varying fluctuation rates corroborate variations in the magnetic anisotropy and exchange interaction dominating the energy barrier between these states, respectively. The persistent switching itself confirms degenerate spin chirality in the amorphous films and particle-like properties of the $N=2$ skyrmions.

The amorphous Fe-Ge thick films $(80 \mathrm{~nm})$ were grown at room temperature on amorphous silicon nitride by coevaporation of Fe and Ge from an electron beam source and an effusion cell, respectively, and capped with a $3 \mathrm{~nm}$-thick aluminum layer. Magnetometry and spectroscopy measurements are carried out with $a-\mathrm{Si}-\mathrm{N}(500 \mathrm{~nm}) /$ $a-\mathrm{SiO}_{x}(30 \mathrm{~nm}) / / \mathrm{Si}$ wafers; commercial $a-\mathrm{Si}-\mathrm{N}(30 \mathrm{~nm})$ membranes are used for Lorentz microscopy and X-ray scattering experiments. Growth rates ranged from 0.25 to $0.35 \AA^{-1}$ depending on the composition; growth temperature was nominally room temperature $\left(T \lesssim 60^{\circ} \mathrm{C}\right)$ and base pressure was below $10^{-8} \mathrm{mbar}$. The film structure was monitored during growth using a reflection high-energy electron diffraction detector and confirmed afterward by X-ray and electron diffraction using a $200 \mathrm{~nm}$-sized probe. As previously discussed, ${ }^{[40]}$ there is significant evidence that $a-\mathrm{Fe}_{x} \mathrm{Ge}_{1-x}$ and $a-\mathrm{Fe}_{x} \mathrm{Si}_{1-x}$ are amorphous for $x<0.67$. Temperature and field-dependent magnetization and magneto-transport measurements versus $x$ for both materials systems are essentially identical and consistent with an amorphous structure approaching the transition from metal to insulator with decreasing Fe concentration. ${ }^{\left[{ }^{40]}\right.}$ The electron diffraction pattern (inset in Figure 1a; additional images in Reference [40]) shows a relatively sharp but continuous ring, which could, in principle, point to very tiny (sub-2 nm) nanocrystals, but it is also consistent with, and commonly observed in, an amorphous structure. Such tiny nanocrystals are generally unstable with 

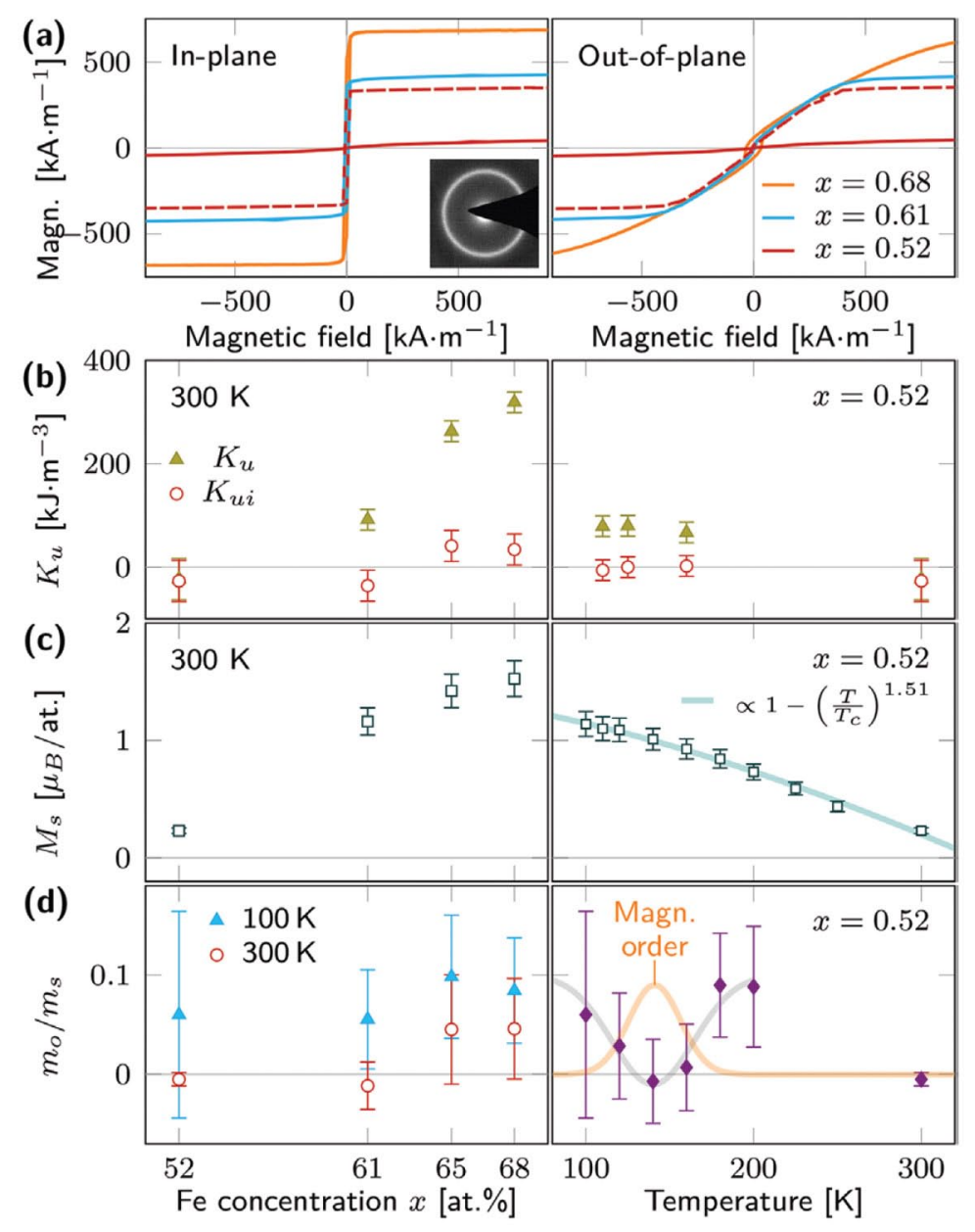

Figure 1. Composition and temperature dependence of magnetic anisotropy and magnetization of amorphous $\mathrm{Fe}_{x} \mathrm{Ge}_{1-x}$ films.

a) In-plane (left) and out-of-plane (right) magnetic hysteresis loops. Solid curves are measured at room temperature; the dashed curves for $x=0.52$ are measured at $110 \mathrm{~K}$. Inset shows electron diffraction pattern recorded after growth confirming amorphicity.

b) Magnetic anisotropy $K u$ versus $x$ at $300 \mathrm{~K}$ (left) and versus $T$ for $x=0.52$ (right) showing in-plane preference for iron-rich samples owing to predominant demagnetization field.

c) Saturation magnetic moment per iron atom retrieved from in-plane $M(H)$ magnetometry data versus $x$ at $300 \mathrm{~K}$ (left) and versus $T$ for $x=0.52$ (right). The temperature dependence of the saturation magnetization is described by the Bloch law with an exponent of $\eta=1.51$.

d) Iron orbital-to-spin moment ratio derived from X-ray magnetic circular dichroism spectra versus $x$ at 100 and $300 \mathrm{~K}$ (left) and versus $T$ for $x=0.52$ (right), exhibiting a significant drop for $x \lessgtr 0.61$ and, for $x=0.52$, an anomalous temperature dependence near $140 \mathrm{~K}$ where the magnetic order of the helical spin phase peaks. The temperature dependence of the Fe orbital-to-spin moment ratio is fitted by a Gaussian, $\max m_{\mathrm{o}} / m_{\mathrm{s}}-\left(T_{\mathrm{c}}=140 K\right)$. 
respect to the amorphous structure, and, more importantly, would have shown up in high-resolution transmission electron microscopy images (which they do for films with $x>0.67$ but not for lower $x$ ). The sharpness of the ring indicates significant short-range order and, perhaps, a non-trivial degree of medium-range order in the amorphous structure. This, in turn, suggests the possibility of a local atomic environment and local DMI similar to B20 FeGe. While there is no net chirality in the amorphous structure, i.e., degenerate spin chirality, it may prevail on the local scale, consistent with observations in the present work. Film composition, homogeneity and atomic density were determined with an uncertainty of \pm 1 at $\%$ from Rutherford backscattering spectra and nanoscale imaging using energy-dispersive X-ray spectroscopy with transmission electron microscopy. ${ }^{[40]}$

The magnetic properties of the deposited films are retrieved from their magnetic hysteresis loops (Figure 1a), which were acquired by superconducting quantum interference device magnetometry and vibrating sample magnetometry for samples with Fe concentration of $x=0.52,0.61$ and $x=0.65,0.68$, respectively. For each sample, the diamagnetic background from substrate and capping layer is subtracted via measuring the sample mass in a microbalance and determining susceptibility of a bare substrate with capping layer. The magnetic anisotropy $K_{\mathrm{u}}$ is calculated via $K_{\mathrm{u}}=\mu_{0} \int_{\|} M \mathrm{~d} H-\mu_{0} \int_{\perp} M \mathrm{~d} H$ of hysteresis-free $M(H)$ data taken with $H$ applied in-plane and out-of-plane, respectively. Subtracting contributions from the demagnetization field leads to the intrinsic magnetic anisotropy $K_{\mathrm{ui}}=K_{\mathrm{u}}-1 / 2 \mu_{0} M_{\mathrm{s}}^{2}$ (Figure $1 \mathrm{~b}$ ) with the saturation magnetization $M_{\mathrm{s}}$ (Figure 1c). The amorphous Fe-Ge films possess an in-plane magnetic anisotropy dominated by the demagnetization field (Figure 1a,b); no indications of a magneto-crystalline anisotropy are observed. The saturation magnetization per Fe atom decreases with decreasing iron concentration $x$, which results in a weaker exchange interaction, and follows the Bloch law ${ }^{[44]}$ for bulk ferromagnets: $M_{\mathrm{s}}(T)=M_{\mathrm{s}}(0)\left(1-\left(T / T_{\mathrm{c}}\right)^{\eta}\right)$ with $\eta=1.51, M_{\mathrm{s}}(0)=$ $1.37 \mu_{\mathrm{B}}$, and $T_{\mathrm{c}}=333 \mathrm{~K}$ for $x=0.52$ (Figure $1 \mathrm{c}$ ). Its $T=0 \mathrm{~K}$ value agrees well with the experimental value measured at $2 \mathrm{~K}$ of $M_{\mathrm{s}}(2 K)=(1.36 \pm$ $0.125) \mu_{\mathrm{B}}$. Considering the slightly larger iron concentration, the measured values are in good agreement with $M_{\mathrm{s}}(0) \approx 1 \mu_{\mathrm{B}}$ per Fe atom in B20 FeGe single-crystals, ${ }^{[45]}$ which suggests a similar short-range order and local atomic environment. The absence of terms with larger exponents $\eta \gtrsim 2$, typically introduced in nanostructures ${ }^{[4-49]}$ to describe the spatial confinement of thermal spin waves reducing the magnetic moment at finite temperatures, ${ }^{\left[{ }^{50]}\right.}$ corroborates the homogeneity of the amorphous films in view of exchange interactions. Small local variations in exchange 
and magnetic anisotropy, whose contributions are averaged out on the micro scale and invisible to integral measurements, are discussed below.

A more detailed analysis of the distribution of the electron orbital orientation, accessible by the net orbital moment, was carried out with XMCD spectroscopy, performed at beamline 6.3.1 at the Advanced Light Source (Berkeley, CA). The X-ray absorption spectra near the Fe $L_{3,2}$ $[(\approx 690-750) \mathrm{eV}]$ edges are recorded while applying an external magnetic field ( $\left.\pm 800 \mathrm{kA} \mathrm{m}^{-1}\right)$, exceeding the saturation fields normal to the sample surface. The spectra are retrieved from the current of electrons emanating from the surface providing a probing depth of about $6 \mathrm{~nm}$. The ratio of orbital $\left(m_{0}\right)$ to spin $\left(m_{s}\right)$ Fe moments is quantified from the spectra integrals ${ }^{[51,52]}$

and

$$
q=\int_{L_{3}+L_{2}}\left(\mu_{+}-\mu_{-}\right) \mathrm{d} \omega
$$

$$
p=\int_{L_{3}}\left(\mu_{+}-\mu_{-}\right) \mathrm{d} \omega
$$

as

$$
m_{\mathrm{o}} / m_{\mathrm{s}}=\left(\frac{4}{3}\right) \frac{q}{(6 p-4 q)}
$$

with an experimental uncertainty dominated by statistical errors of consecutive spectra (Figure S2, Supporting Information). The spin and orbital moments retrieved from XMCD represent quantities per atom averaged over the entire sample. Focusing on the orbital-to-spin moment ratio instead of quantifying spin and orbital moments separately avoids contributions from experimental uncertainties related to the electron hole density on the order of $\approx 20-30 \%$, volume density and X-ray polarization of the used bending magnet. In fact, a comparison between magnetic moment retrieved from magnetometry and XMCD reveals a lower magnetic moment for the XMCD data (Figure S3, Supporting Information), which may be due to an overestimated volume density and/or degree of polarization of X-rays generated by the bending magnet or underestimation of the electron hole density. On the other hand, a change in the orbital moment without correlation with the spin moment is insufficient as the material could lose magnetism altogether. A typical reduction of the exchange interaction with increasing temperature or decreasing concentration of magnetic elements decreases both spin and orbital moment, and preserves their ratio. A high degree of orbital alignment 
manifests a large orbital moment as reported in $L 1_{0}$ FePt. ${ }^{[53,54]}$ We observe a significant drop of the orbital-to-spin-moment ratio both as a function of Fe concentration ( $x \lesssim 0.61$ ) and temperature, for example, near 140 $\mathrm{K}$ for $x=0.52$ (Figure $1 \mathrm{~d}$ ). As shown below, the suppression of the orbital moment coincides with the emergence of non-collinear helical spin textures. These observations indicate the necessity of disordered electron orbitals for a random DMI stabilizing chiral spins and refute a possible argument about dipole-stabilized spin textures existent in multilayer stacks with perpendicular magnetic anisotropy.

To further examine the impact of orbital disorder on the microscopic magnetization configuration, we visualize the in-plane magnetic induction employing Lorentz microscopy with exit wave reconstruction. ${ }^{[30]}$ Lorentz microscopy was carried out using a $300 \mathrm{keV}$ aberration-corrected transmission electron microscope (TEAM I) with a Gatan K2-IS direct electron detector operated in electron-counting mode (pixel size at 2000x: $\approx 0.45 \mathrm{~nm}$ ) at the Molecular Foundry (Berkeley, CA). The electron intensity was acquired at various focal planes $\Delta f_{\mathrm{k}}=(\approx 0$ to -5$) \mathrm{mm}$ at $5 \mathrm{~Hz}$ over $6 \mathrm{~s}$ and aligned via phase auto-correlation for each plane individually. The samples are mounted onto a cryo holder with two orthogonal tilt axes and cooled with a cold finger using liquid nitrogen; measurements are performed after temperature stabilization. Further details are given in the Supporting Information.

The electron phase shift $\phi$ of a coherent, originally planar electron wave front, accumulated due to interaction with electromagnetic fields, is retrieved from $\gtrsim 10$ focal planes using an iterative exit wave reconstruction, ${ }^{[30]}$ known as Gerchberg-Saxton algorithm. ${ }^{[55]}$ This approach significantly enhances spatial resolution and sensitivity compared with the transport-of-intensity (TIE) ansatz, ${ }^{[56]}$ and is essential to properly reconstruct the electron phase of amorphous materials exhibiting structural voids and grains with their own polar phase contrast $\phi_{\mathrm{e}}$ disturbing magnetic contributions. In reciprocal space, the magnetic contribution to the electron phase $\phi$ is given by: ${ }^{[57]}$

$$
\phi_{m}\left(q_{x}, q_{y}\right)=\frac{i \pi \mu_{0} M_{s} t}{\Phi_{0}} \cdot \frac{m_{x} q_{y}-m_{y} q_{x}}{q_{x}^{2}+q_{y}^{2}}
$$

with the unit magnetization vector components $m_{x, y}$ perpendicular to the electron propagation direction $z$, and reciprocal unit vector components 
$q_{x, y} . \Phi_{0}, M_{s}$, and $t$ are magnetic flux quantum, saturation magnetization, and Fe-Ge film thickness, respectively. Experimentally, the in-plane components of the magnetic induction are derived from the 2D gradient of the electron phase taking advantage of the Aharonov-Bohm effect and Stokes' theorem:

$$
\nabla \phi=\nabla\left(\phi_{\mathrm{e}}+\phi_{\mathrm{m}}\right) \approx \nabla \phi_{\mathrm{m}}=\frac{\pi}{\Phi_{\mathrm{o}}} t\left(B_{y^{\prime}}-B_{x}, 0\right)
$$

This approximation takes into account different length scales and phase amplitudes of electrostatic $\left(\phi_{\mathrm{e}}\right)$ and magnetic $\left(\phi_{\mathrm{m}}\right)$ contributions as well as the slow convergence of low-frequency components (large, non-electrostatic features) during the iterative phase retrieval. ${ }^{[58]}$ The inplane components of the magnetization are presented as arrows of a 2D vector field with color indicating the in-plane orientation (Figure 2). The corresponding electron phases are displayed as background. Both quantities are separately shown in Figures S2 and S3, Supporting Information.

$\mathrm{Fe}_{x} \mathrm{Ge}_{1-x}$ films with $x \gtrsim 0.63$ are ferromagnetic at room temperature and exhibit a weak local modulation of the in-plane magnetization, typically referred to as magnetization ripples (Figure $2 \mathrm{a}$ ). The opening angle of the ripples is $\approx 10^{\circ}$ and determined by correlating electron wave propagation simulations of various spin textures with the experimental contrast in the vicinity of metastable $180^{\circ}$ domain walls that generate interference fringes (Figures S8 and S9, Supporting Information). This way, experimental limitations, typically inherent to imaging techniques, are overcome, namely access to only the relative change within the field of view and lack of information about lowest-frequency components, that is, constant offsets of the magnetic induction.

Reducing the iron concentration to $x \lesssim 0.63$ weakens the exchange interaction and transforms the magnetization ripples pattern into extended striped domain patterns with noticeably larger contrast and varying degree of disorder, which are reconstructed as helical spins (Figure $2 \mathrm{~b}, \mathrm{c}$ ). Samples with an iron concentration of $x \approx 0.61$ show a globally indefinite lattice orientation, that can vary continuously on the micro scale in the form of, for example, a full $180^{\circ}$ rotation (Figure $\mathrm{S} 10$ and S11, Supporting Information) or spin dislocations. At remanence, a fractal-like intensity distribution appears that vanishes in a normal magnetic field simultaneously enlarging the striped contrast and locally generating W-shaped zig-zag walls (Figure S10, Supporting Information). 

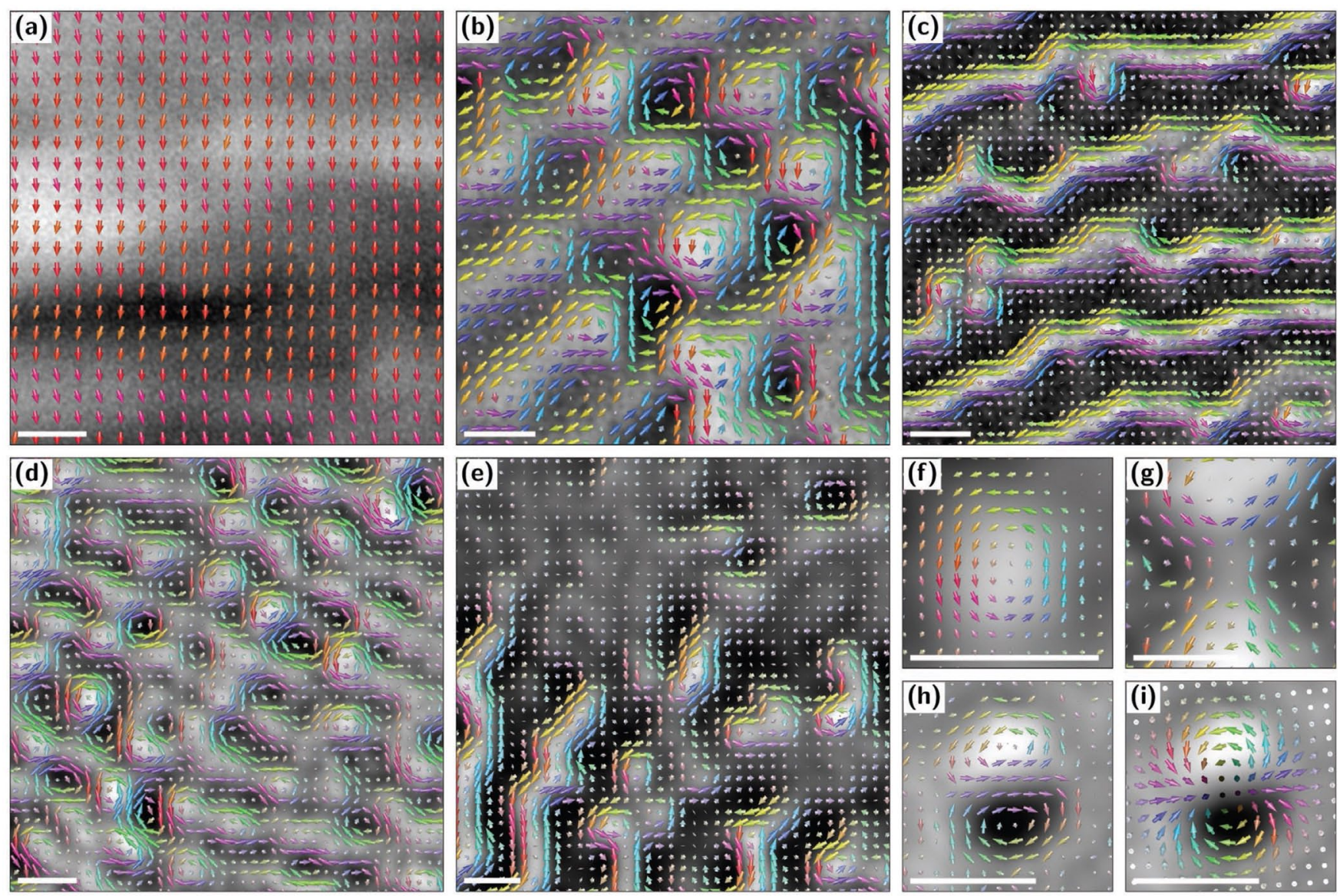

Figure 2. Chiral spin textures in amorphous $\mathrm{Fe}_{x} \mathrm{Ge}_{1-x}$ thick films with random DMI visualized by high-resolution Lorentz microscopy with exit wave reconstruction. Color and direction of arrows indicate in-plane component of magnetization vector field retrieved as 2D gradient from the reconstructed electron phase, shown as background (contrast is scaled to provide best visibility).

a) Magnetization ripples ( $\left.x=0.65, T=300 \mathrm{~K}, H=0 \mathrm{kA} \mathrm{m}^{-1}\right)$.

b) Helical spins decorated with Bloch-type skyrmions $\left(x=0.61, T=300 \mathrm{~K}, H=32 \mathrm{kA} \mathrm{m}^{-1}\right)$.

c) Disordered helical spins ( $\left.x=0.52, T=155 \mathrm{~K}, H=0 \mathrm{kA} \mathrm{m}^{-1}\right)$.

d) Skyrmion glass $\left(x=0.52, T=155 \mathrm{~K}, H=32 \mathrm{kA} \mathrm{m}^{-1}\right)$.

e) Coexistence of helical spins and isolated skyrmions near phase transition $(x=0.52$, $T=135 \mathrm{~K}, H=0 \mathrm{kA} \mathrm{m}^{-1}$ ).

f) Isotropic Bloch skyrmion ( $\left.x=0.52, T=135 \mathrm{~K}, H=0 \mathrm{kA} \mathrm{m}^{-1}\right)$.

g) Isolated antiskyrmion $\left(x=0.52, T=155 \mathrm{~K}, H=64 \mathrm{kA} \mathrm{m}^{-1}\right)$.

h) Anisotropic skyrmion with dipolar electron phase contrast $(x=0.52, T=110 \mathrm{~K}$, $H=0 \mathrm{kA} \mathrm{m}^{-1}$ ).

i) Simulated electron phase contrast of $N=2$ skyrmion closely matches the experimentally observed dipolar contrast falsely reconstructed as a biskyrmion configuration (h).

The apparent asymmetry in "up" and "down" regions in (c) is due to using TIE, and the fact that divergent "domain walls" appear larger than convergent ones in Lorentz microscopy. Scale bar in each panel is $100 \mathrm{~nm}$. 
Contrary to common (closed) zig-zag walls, ${ }^{[59]}$ separating magnetic domains with in-plane magnetization pointing toward or away from the walls, the present open specimens cannot be incorporated into in-plane magnetized domains. Such an arrangement would require a non-vanishing easy axis (uniaxial in-plane magnetic anisotropy) and diamond-like shape. Moreover, the striped domain pattern would be absent, weaker or less ordered, similar to the magnetization ripples, and the domain wall contrast were much larger. This applies also to a possible conical spin formation in the presence of a normal magnetic field (Figure S10d,e, Supporting Information). If the modulation were parallel to the net magnetization, the zig-zag domain wall configuration would be unstable; no contrast would appear if the modulation were perpendicular to the net magnetization. Instead, considering that Lorentz microscopy probes the in-plane magnetic induction rather than the in-plane magnetization, the zig-zag wall contrast and, to some extent, the fractal-like contrast can be explained by a changing lattice orientation of the helical spins that generates a magnetization divergence and stray fields. Such a contrast was specifically visualized with magnetic force microscopy in B20 FeGe crystals at helix lattice boundaries ${ }^{[60]}$ that lack a net in-plane magnetization.

These observations reveal the general trend of the magnetization configurations in the amorphous iron germanium samples (Figure 3a). Lowering the iron concentration from $x \gtrsim 0.63$ to $x \approx 0.52$ transforms a ferromagnetic system into a proper helical spin system with reduced magnetic exchange interactions. This transition is mediated by helical spins with a potential remanent in-plane magnetization that vanishes in the presence of a normal magnetic field $(x \approx 0.61)$. We stress that the existence and continuous transformation of non-collinear spin textures in soft-magnetic films without magnetic anisotropy refutes the argument of a possible dipole-driven formation emerging in multilayer stacks with perpendicular magnetic anisotropy. In addition, we observe topological magnetization vector fields, namely skyrmions with different topological charge $N$ and both spin circulations. The latter refers to the sense of rotation of the in-plane magnetization. At and near remanence, the magnetization configuration is dominated by isotropic Bloch skyrmions $(N=$ 1) (Figure 2f) decorating helical spins (Figure 2b,c). Aside from small areas of closely packed skyrmions with both spin circulations, no extended skyrmion lattices are observed (Figure 2b); the location and periodicity of the overwhelming majority of skyrmions coincides with the helix 

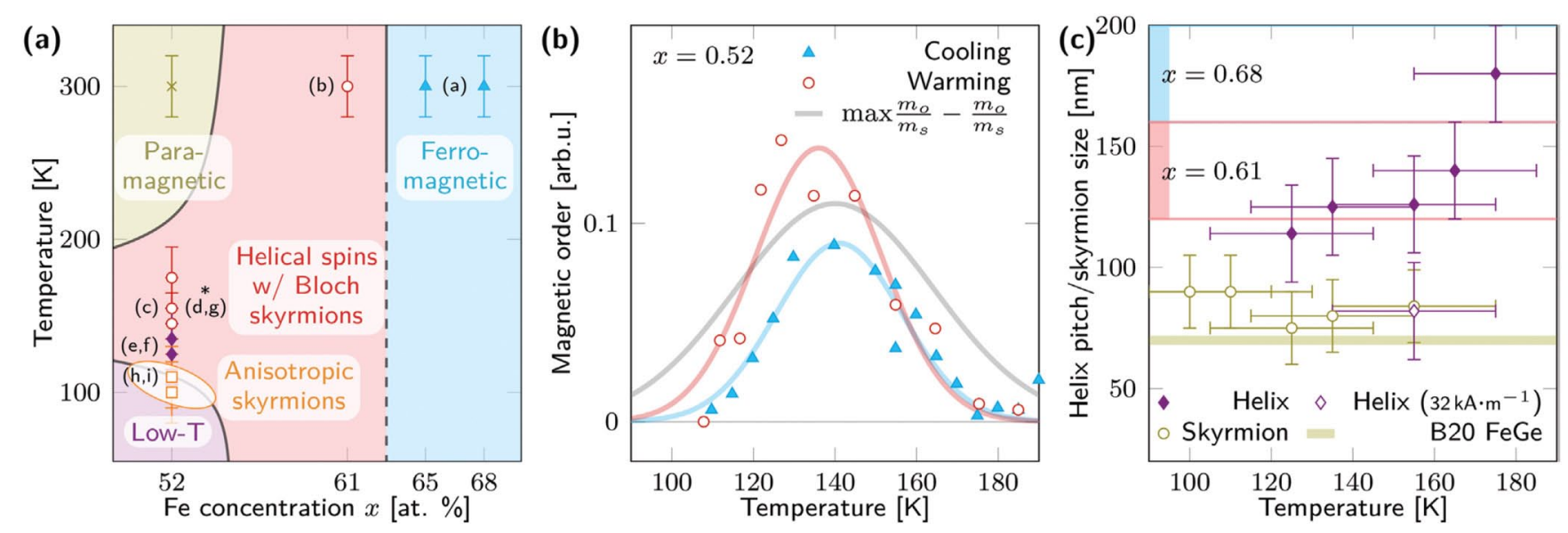

Figure 3. Magnetic phase diagram and long-range order of non-collinear spin textures. a) Experimental magnetic phase diagram assembled from magnetization configurations shown in Figure 2, illustrating transition from ferromagnetic to non-collinear, topological states with decreasing Fe concentration which causes a reduced Heisenberg exchange interaction. The low-temperature phase lacks with exception of local spin excitations, both long-range and short-range magnetic order. Solid and dashed lines serve as a guide to the eye. b) Magnetic order of helical phase ( $x=0.52)$ for warming and cooling cycle quantified by intensity of magnetic Bragg peak. Error bars $( \pm 20, \pm 0.01 \mathrm{~K}$ ) are omitted for visibility. The red and blue curves are Gaussian fits centered around $140 \mathrm{~K}$ that serve as a guide to the eye and coincide with the suppression of the orbital-to-spin moment ratio extracted from Figure 1d. c) Helical spin pitch and skyrmion size in $x=0.52$ samples at remanence and temperatures from 100 to $200 \mathrm{~K}$. Helix periodicity in out-of-plane bias field is plotted as individual data point at $155 \mathrm{~K}$. Room temperature values for helix $(x=0.61)$ and ripples $(x=0.68)$ periodicity are displayed as horizontal bars. Periodicity of helical spins and skyrmion lattice $(70 \mathrm{~nm})$ in B20 FeGe single-crystals is shown for reference.

symmetry and is not determined by structural imperfections (pinning sites). In $x=0.52$ samples, the disordered helical spins (Figure 2c) transition in a magnetic bias field into a skyrmion glass-like state with disordered Bloch skyrmions (Figure 2d) and occasionally isolated antiskyrmions $(N=-1)$ (Figure 2g); $N=2$ skyrmions appear as isolated states at low temperature and remanence (Figure $2 \mathrm{~h}, \mathrm{i}$ ). These experimental observations are summarized in a phase diagram (Figure $3 a$ ). The formation of chiral topological states in $x \lesssim 0.63$ samples stems from a reduced Heisenberg exchange interaction, smaller saturation magnetization, and an effectively enhanced local DMI as suggested by the reduced 
orbital moment (Figure 1d). Despite being qualitatively sound, it is fascinating considering the lateral extent of skyrmions and helical spins is two orders of magnitude larger than the length scale on which local DMI varies, which should cause a substantial cancellation. Our current interpretation is based on a non-vanishing, sizable, random exchange interaction that leads to vector spin frustration and favors non-collinear spins and degenerate spin chirality similar to frustrated dipole spin systems with structural disorder and emergent chiral vortex lattices. ${ }^{[41-43]}$

The relation between electron phase and magnetic induction, given by Equation (2), is ambiguous, which becomes critical when visualizing anisotropic topological states. To examine this aspect, we approximate skyrmions as 2D spin textures with a depth-independent profile defined in polar coordinates $(r, \phi)$ as

$$
\mathbf{m}=(\sin \Theta(r) \cos \Phi(\phi), \sin \Theta(r) \sin \Phi(\phi), P \cos \Phi(r))
$$

and use the topological charge $N$, chirality $C$, polarity $P$, and $\Phi(\phi)=N(\phi+C) \cdot \Theta(r)$, which is a radial function that determines the core size, similar to half the helix periodicity, and depends on the preferred canting angle. Note that the small twist of 3D skyrmions tubes ${ }^{[61]}$ does merely affect the amplitude but not the symmetry and shape of the phase shift; the latter is crucial to the identification. The electron phases corresponding to these magnetization vector fields are calculated according to Equation (1).

While isotropic Bloch-type skyrmions $(N=1, C= \pm \pi / 2)$ cause a polar phase contrast and a lateral extent similar to half the corresponding helix pitch (Figure 2b,c), Néel-type skyrmions are (like Néel domain walls) invisible to Lorentz microscopy due to electron deflection along the circumference. ${ }^{\left[{ }^{30]}\right.}$ Higher-order topological states, such as $N=2$ skyrmions, are anisotropic solitary spin textures with a dipolar (for $N>2$, multipolar) phase contrast and possess both Bloch and Néel character (Figure 2i). A similar phase contrast belongs to bound pairs of skyrmions with opposite chirality and $N=0$, referred to as biskyrmions (Figure $2 \mathrm{~h}$ ). In fact, the reconstruction of the in-plane magnetic induction/magnetization from either state's dipolar phase contrast leads to a biskyrmion configuration (Figure S16, Supporting Information) due to nearly identical phase contrasts. While the dipolar contrast has traditionally been assigned to biskyrmions, ${ }^{[3,36,62]}$ a careful analysis of Lorentz microscopy data with the TIE ansatz ${ }^{[63,64]}$ or, as done here, with 
exit wave reconstruction suggests alternative interpretations. This includes Bloch-type bubbles with two Bloch lines, ${ }^{[63,64]}$ Bloch skyrmions appearing as biskyrmions due to phase offset, ${ }^{[62]}$ and $N=2$ skyrmions (Figure 2h,i). Indeed, modeling of biskyrmions reveals a highly anisotropic lateral extent, ${ }^{[65]}$ typically absent in the experimental data, and an irreproducibility in micromagnetic simulations. Bloch-type bubbles with two Bloch lines, each pinned on two defects on opposite sites of the bubbles, and tilted Bloch or Néel skyrmions can also be excluded due to distinct, more confined electron diffraction and phase contrast, and an exclusive observation of dipolar and polar phase contrast in the low- and high-temperature phase, respectively. The isotropic lateral extent of the dipolar phase contrast (Figure $2 \mathrm{i}$ ) and the magnetization dynamics discussed below corroborate the conclusion of stabilized $N=2$ skyrmions in the present case.

The reduced exchange interaction in the $x=0.52$ sample causes a rich temperature dependence of the magnetic properties, absent in specimens with larger iron concentration. We study the magnetic order near the temperature-driven phase transition from helical phase to low-temperature phase with Lorentz microscopy and resonant coherent X-ray scattering. The magnetic induction is approximated from a time series recorded at a constant focal plane $(\Delta f=-3.9 \mathrm{~mm})$ with 5 or $10 \mathrm{~Hz}$ over $60 \mathrm{~s}$ using the TIE equation ansatz ${ }^{[56]}$ :

$$
\frac{-2 \pi}{\lambda I_{0} \Delta f}\left(I-I_{0}\right)=\nabla^{2} \phi
$$

and Equation (2). Note that this approach is accurate only for small $\Delta f$ and negligible changes in $I$, that is, $I \simeq I_{0}{ }^{\left[{ }^{[56]}\right.}$ X-ray scattering was performed at the COSMIC scattering beamline at the Advanced Light Source. The magnetic diffraction intensities were recorded at the Fe $L_{3}$ absorption edge and at normal incidence with a $7 \mu \mathrm{m}$ pinhole aperture to provide coherent X-rays. Coherent X-rays diffracted from nano scale modulations create an interference pattern (speckle) under the magnetic diffraction peak, which is related to the Fourier transform of the magnetization distribution. Hence, it probes the spatial modulation of the out-of-plane magnetization component on the nano scale with a temporal resolution of $0.6 \mathrm{~s}$.

The system becomes magnetically ordered at remanence between 100 and $200 \mathrm{~K}$ (Figure 3b) to form helical spin textures. The long-range order of the non-collinear spin texture is defined as the intensity of the 

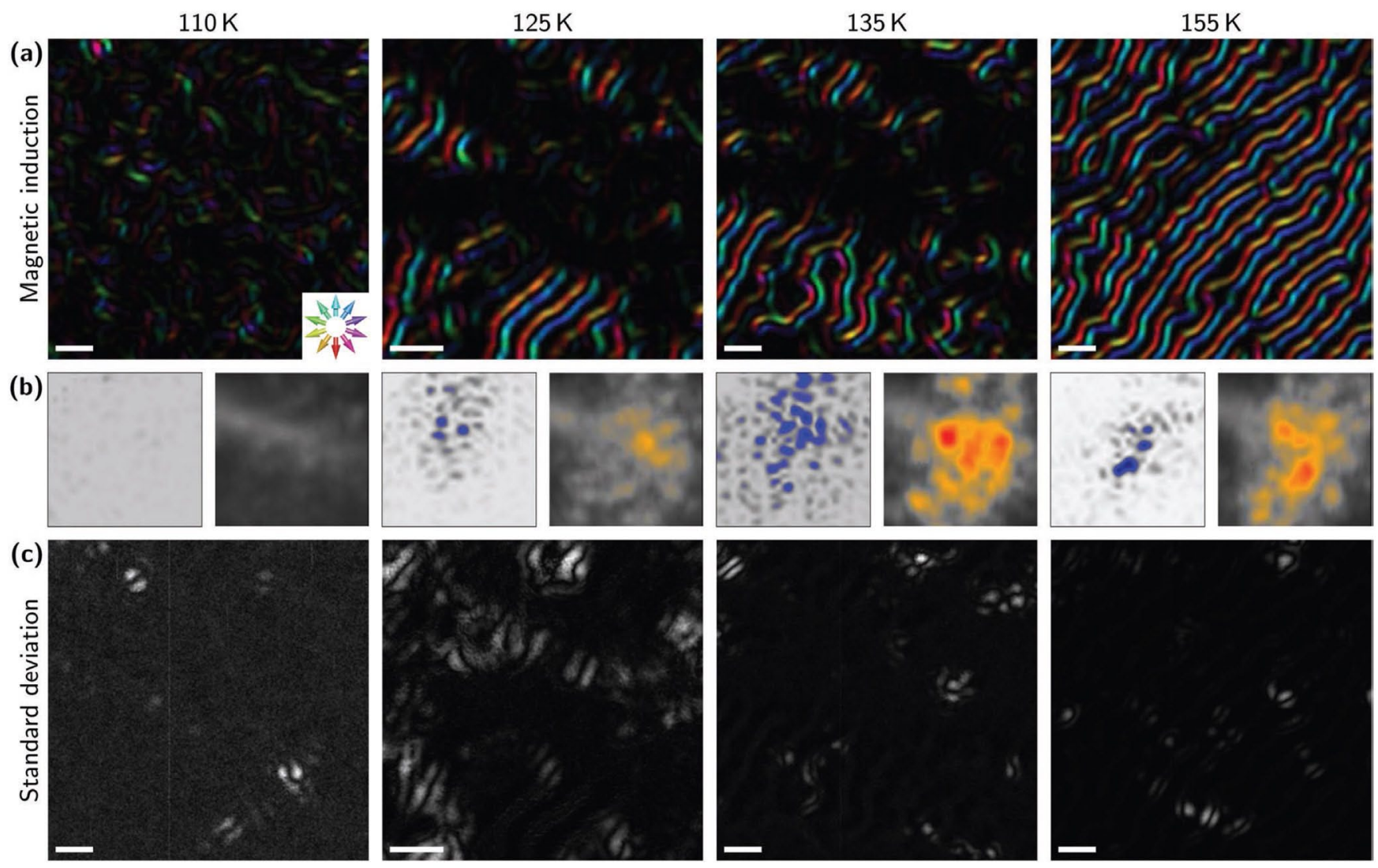

Figure 4. Magnetization configurations and thermal spin fluctuations near magnetic phase transition at remanence $(x=0.52)$. a) Magnetic induction from Lorentz microscopy with TIE revealing phase transition from disordered helical phase ( $\gtrsim 155 \mathrm{~K})$ to low-temperature phase, lacking both short-range and long-range magnetic order $(\lesssim$ 110 K). b)(left) Quadrant of Fourier transform of (a) and b)(right) speckle pattern near magnetic Bragg peak obtained with resonant coherent X-ray scattering displaying similar patterns (order) for in-plane and out-of-plane magnetization components, respectively. c) Standard deviation of time series corresponding to (a) displays location and amplitude of spin fluctuations. Scale bar in each panel is $200 \mathrm{~nm}$.

magnetic Bragg peak and coincides almost perfectly with the suppression of the orbital-to-spin moment ratio. The upper boundary representing the Curie temperature refers to the phase transition between paramagnetic/weak ferromagnetic state and helical phase with isolated Bloch-type skyrmions (Figures 3 and 4a). Below $130 \mathrm{~K}$, the helical spin configuration breaks up and transitions into a low-temperature phase without obvious non-collinear magnetic order (Figure 4a) except for isolated $N=2$ skyrmions, as discussed below. X-ray speckle patterns resemble the Fourier transform of the Lorentz microscopy data not only 
in shape and intensity, but also in temperature dependence (Figure $4 \mathrm{~b}$ and Figure S15, Supporting Information). This implies that both in-plane and out-of-plane magnetization components lose long-range and shortrange order on the experimentally accessible $0.1 \mathrm{~s}$ time scale. We note that magnetometry and XMCD spectroscopy are measured in an external magnetic bias field and show a non-vanishing magnetic moment below the phase transition. This suggests that either the spin fluctuation rate at remanence exceeds the temporal resolution of both Lorentz microscope and scattering experiment, and washes out the magnetic contrast, or the feature size abruptly decreases below the spatial resolution limit. Since both spin moment and anisotropy change only slightly, we attribute this phase transition to an abrupt variation in spin-orbit coupling and spin frustration, which is not uncommon among helimagnets and spin frustrated materials. Determining the physical origin of this phase transition is subject to ongoing studies as it does not affect the outcome of this work.

The periodicity of the helical spins decreases with decreasing temperature to values less than the room temperature periodicity of helical spins $(x=0.61)$ and magnetization ripples $(x=0.68)$, and asymptotically approaches $100 \mathrm{~nm}$ (Figure 3c). In an out-of-plane magnetic field, the helix pitch further reduces to the size of a skyrmion, which is $75 \pm 15 \mathrm{~nm}$ and within statistical uncertainty temperature and magnetic field independent. The skyrmion size is defined as the mean diameter of the circumferential in-plane magnetization (Figure 2f), and agrees well with the periodicity of skyrmion lattices in B20 FeGe single-crystals of $70 \mathrm{~nm} .{ }^{[23]}$ The helical spins possess a sizable disorder apparent in both real space (Figure 4a) and speckle patterns of in-plane (Figure 4b(left)) and out-of-plane (Figure 4b(right)) magnetization components. The disorder is caused by a combination of 1) an actual discontinuity in the helical spin configuration in form of spin dislocations, 2) skyrmions decorating the spin helix, and 3) deformations due to structural defects and local variations in the magnetic anisotropy. The latter contribution is evident from the location and amplitude of thermal spin fluctuations, which is presented in form of the standard deviation of the time series recorded at $10 \mathrm{~Hz}$ over $60 \mathrm{~s}$ (Figure 4c). Hence, the analysis of thermal fluctuations is restricted to recurring events. While spatially correlated fluctuations reach a maximum near the phase transition at domain boundaries, including those separating discontinuous regions 
of helical spins and isolated skyrmions, they persist at lower pace well beyond the transition temperature. This behavior is closely related to the disorder of the helical spins and the corresponding deviation from a sinusoidal magnetization distribution with a constant canting angle between adjacent spins. The incongruence promotes localized thermal spin fluctuations in form of a persistent advancement and retreat back to the original location, observed in the time series, that are spatially confined to half the helix periodicity, or the size of a skyrmion, and appear as dipolar contrast in the standard deviation.

Although the low-temperature phase shows almost no magnetic contrast (Figure 4a) and lacks long-range order (Figure 3b), thermal spin fluctuations prompt a dipolar contrast in the standard deviation representative of local spin excitations (Figure $4 \mathrm{~b}$ and Figure S18, Supporting Information). These fluctuations signify a frequent switching of $N=2$ skyrmions between two discrete states, that is, $S= \pm 1$, with a shared uniaxial symmetry axis (Figure 5a). The magnetization configurations $S=1$ and $S=-1$, referring to $N=2$ skyrmions with chirality $C=0$ and $C=\_2$, respectively, are analytically modeled using Equation (3); the corresponding electron phase is calculated according to Equation (1) and reveals excellent agreement with the experimental data (Figure 5a). Mathematically, the chirality $C$ is an improper parameter to describe anisotropic magnetic solitons since it merely represents an azimuthal rotation. However, in real materials, the orientation of these anisotropic states ( $S=1$ vs. $S=-1$ ) and the local spin chirality between adjacent atoms matter due to different neighboring spins (magnetic environment), local variations in structural, chemical and magnetic properties defining exchange interaction and spin frustration, as well as distinct spin-transfer torque and topological Hall effect upon current excitation. This becomes obvious when following the spins along a straight line through the center of the $N=2$ skyrmion; the spin chain is chiral for any angle and exhibits the opposite spin chirality for $S=1$ and $S=-1$ states.

Observing a persistent switching between these states corroborates a degenerate spin chirality and particle-like properties, and refutes a possible biskyrmion configuration or Bloch-type bubbles with two Bloch lines. The Bloch lines would have to be pinned on two defects on opposite sites of the bubbles and unpinned/switched at the very same time, while existing exclusively in the low-temperature phase. The preferred 

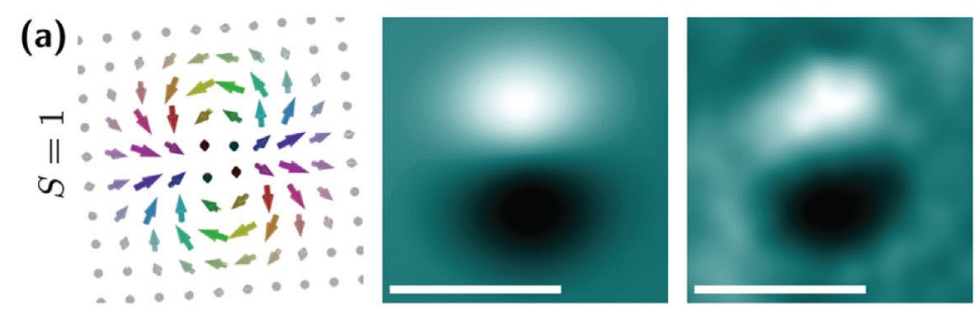

Magnetization

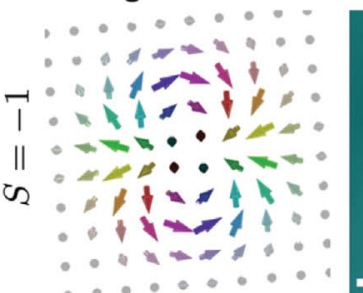

Mod. phase

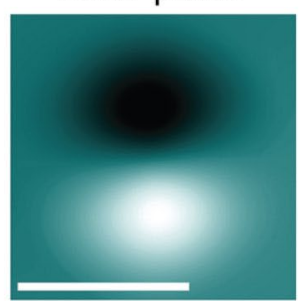

Exp. phase
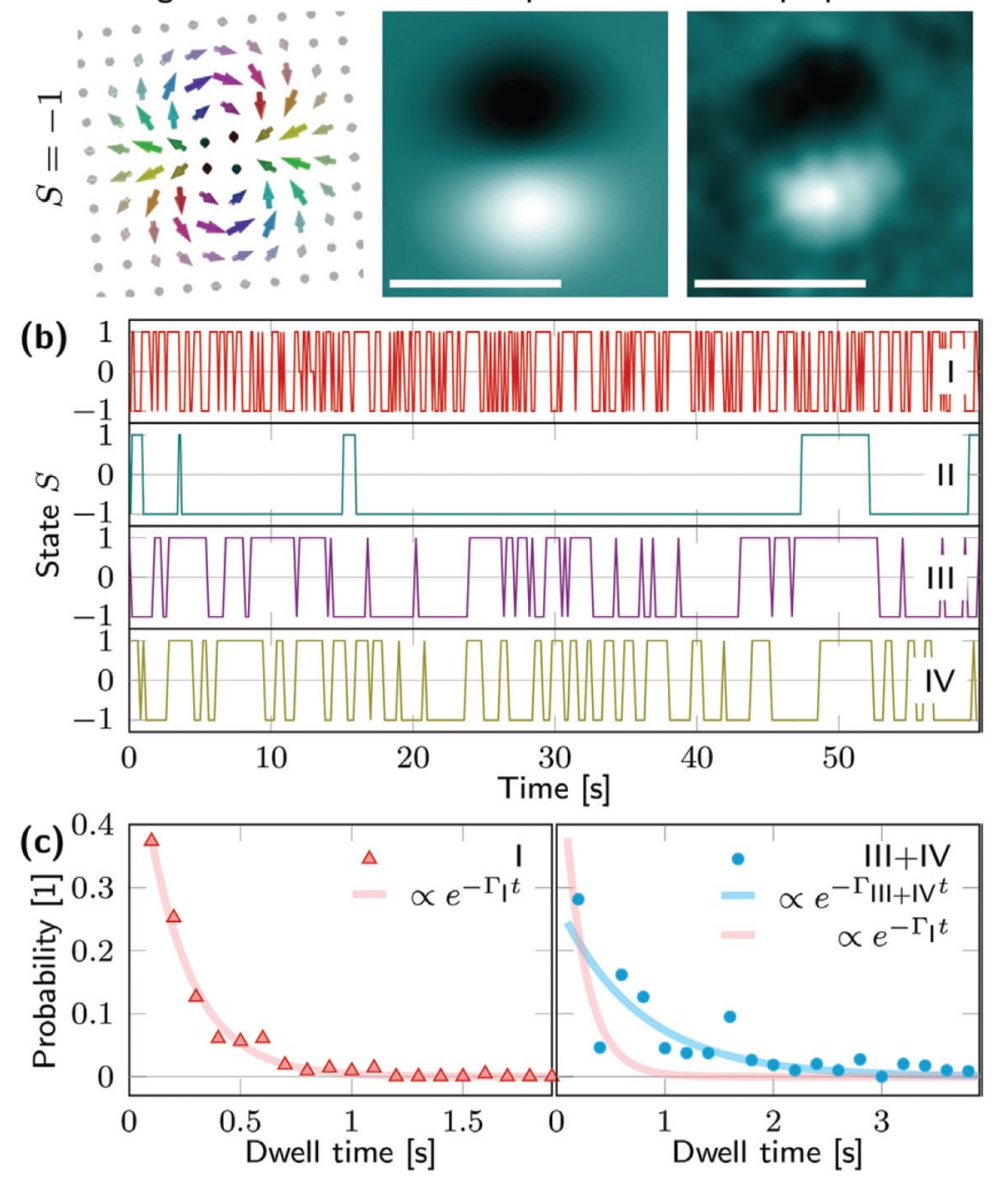

Figure 5. Switching of isolated $N=2$ skyrmions at $110 \mathrm{~K}(x=0.52)$ visualized with Lorentz microscopy.

a) Modeled and experimental electron phase of magnetization configurations $S=1$ and $S=-1$. Experimental data is retrieved from electron intensity averaged over $0.1 \mathrm{~s}$ at focal plane $\Delta f=-3.9 \mathrm{~mm}$. Scale bar in each panel is $100 \mathrm{~nm}$.

b) Temporal evolution of skyrmion configuration $S$ revealing fluctuation rates ranging from $10 \mathrm{~Hz}$ to $10 \mathrm{mHz}$ depending on locations: I, II, III, and IV. Experimental data shown in (a) is taken at location I.

c) Probability of dwell times for location I (left) and sum of locations III and IV (right). No distribution is given for location II due to limited number of switching events. The distributions are fitted with an exponential function leading to $\Gamma_{\mathrm{I}}=4.9 \mathrm{~Hz}$ and $\Gamma_{\mathrm{III}+\mathrm{IV}}=1.3 \mathrm{~Hz}$. 
orientation of the symmetry axis of individual $N=2$ skyrmions, selecting two discrete states, indicates a small local magnetic anisotropy that vanishes on the global scale. The temporal evolution of the magnetization configuration, shown in Figure 5b for four different locations I, II, III, and IV, demonstrates an isothermal fluctuation rate ranging from 10 $\mathrm{Hz}$ to $10 \mathrm{mHz}$ depending on the local environment. This variation is not related to the orientation of the symmetry axis that, too, varies on the nanometer scale (Figure 4c and Figure S18, Supporting Information). The dwell times are evaluated in terms of their probability with a binning size of 0.1 and $0.2 \mathrm{~s}$ for 10 and $5 \mathrm{~Hz}$ data, respectively. Figure 5c plots the probability for location I, and locations III and IV taking advantage of similar fluctuation rates. The probability is fitted with an exponential function $\propto e^{-\Gamma_{j t}}$ to assess the energy barrier $E_{\mathrm{B}}^{j}$ separating configurations $S=1$ and $S=-1$ via the relation

$$
\Gamma_{j}=v e^{-\frac{E_{\mathrm{B}}^{j}}{k_{\mathrm{B}} T}}
$$

The attempt frequency $v$ describes the physical interaction, which we presume as similar throughout the sample. The wide range of fluctuation rates translates into $\Gamma_{\mathrm{I}}=4.9 \mathrm{~Hz}$ and $\Gamma_{\mathrm{III}+\mathrm{IV}}=1.3 \mathrm{~Hz}$ for the depicted probabilities. The difference between the energy barrier at location I and locations III/IV is $12.6 \pm 2.2 \mathrm{meV}$, and can reach up to $\Delta E_{\mathrm{B}}^{j} \gtrsim 60 \mathrm{meV}$ considering $\Gamma_{\mathrm{II}} \approx 10 \mathrm{mHz}$. Even without quantification of the energy barrier, the broad distributions of the energy barrier is evident and demonstrates local variations in the structural and chemical short-range order affecting magnetic exchange interactions and magnetic anisotropy in the present amorphous materials.

We synthesized amorphous $\mathrm{Fe}_{x} \mathrm{Ge}_{1-x}$ thick films that exhibit a shortrange order similar to B20 FeGe single-crystals despite lacking a welldefined symmetry. The suppression of a net orbital moment in samples with $0.52 \lesssim x \lesssim 0.61$, indicating a high degree of electron orbital disorder, was accompanied by the formation of chiral spin textures, such as helical spins, isotropic Bloch-type skyrmions $(N=1)$, and isolated anisotropic magnetization vector fields. In $x=0.52$ samples, we observed a phase transition from a high-temperature helical phase to a low-temperature state, which lacks, with the exception of localized spin excitations, both long-range and short-range magnetic order; spin excitations appear in form of isolated antiskyrmions $(N=-1)$ and $N=2$ skyrmions. Monitoring the temporal evolution of thermal spin fluctuations divulged 
a persistent switching of $N=2$ skyrmions between two discrete states that affirmed degenerate spin chirality, particle-like properties, and a sizable variation in both exchange interaction and magnetic anisotropy. These intriguing experimental results stimulate in-depth investigations of germanium-rich samples with weaker exchange interaction and potentially enhanced spin frustration and dynamics, which had not been accessible due to temperature constraints of experimental tools. Our findings showcase the stabilization of 3D non-collinear spin textures in a structurally and chemically disordered material, which is a milestone toward harnessing theoretically predicted higher-order, anisotropic topological states for novel microelectronics and sensing applications based on magnetic quantum materials. Amorphicity by itself offers additional benefits ranging from a greater flexibility in materials synthesis of multifunctional materials, as they do not require special substrates and growth conditions, to larger Hall effects and hence a higher sensitivity to voltage and strain manipulation, which are promising alternatives to current control.

Acknowledgments This work was funded by the U.S. Department of Energy, Office of Science, Basic Energy Sciences, Materials Sciences and Engineering Division under Contract No. DE-AC02-05-CH11231 within the NEMM program (MSMAG). Work at the Molecular Foundry was supported by the U.S. Department of Energy, Office of Science, Basic Energy Sciences under Contract No. DE-AC02-05-CH11231. This research used resources of the Advanced Light Source, which is a DOE Office of Science User Facility under contract no. DE-AC02-05-CH11231.

Conflict of Interest The authors declare that there are no conflicts of interest.

Supporting Information Supporting Information file (11 MB) is attached.

\section{References}

1] U. K. Rößler, A. N. Bogdanov, C. Pfleiderer, Nature 2006, 442, 797.

2 ] S. Seki, X. Z. Yu, S. Ishiwata, Y. Tokura, Science 2012, 336, 198.

3 ] N. Nagaosa, Y. Tokura, Nat. Nanotechnol. 2013, 8, 899.

4] R. Wiesendanger, Nat. Rev. 2016, 1, 16044.

5 ] L. Wang, Q. Feng, Y. Kim, R. Kim, K. H. Lee, S. D. Pollard, Y. J. Shin, H. Zhou, W. Peng, D. Lee, W. Meng, H. Yang, J. H. Han, M. Kim, Q. Lu, T. W. Noh, Nat. Mater. 2018, 17, 1087. 
6 ] W. Jiang, P. Upadhyaya, W. Zhang, G. Yu, M. B. Jungfleisch, F. Y. Fradin, J. E. Pearson, Y. Tserkovnyak, K. L. Wang, O. Heinonen, S. G. E. te Velthuis, A. Hoffmann, Science 2015, 349, 283.

7 ] S. Woo, K. Litzius, B. Kruger, M.-Y. Im, L. Caretta, K. Richter, M. Mann, A. Krone, R. M. Reeve, M. Weigand, P. Agrawal, I. Lemesh, M.-A. Mawass, P. Fischer, M. Klaui, G. S. D. Beach, Nat. Mater. 2016, 15, 501.

8 ] C. Moreau-Luchaire, C. Moutafis, N. Reyren, J. Sampaio, C. A. F. Vaz, N. Van Horne, K. Bouzehouane, K. Garcia, C. Deranlot, P. Warnicke, P. Wohlhüter, J.-M. George, M. Weigand, J. Raabe, V. Cros, A. Fert, Nat. Nanotechnol. 2016, 11, 444.

9] A. Soumyanarayanan, M. Raju, A. L. Gonzalez Oyarce, A. K. C. Tan, M.-Y. Im, A. P. Petrović, P. Ho, K. H. Khoo, M. Tran, C. K. Gan, F. Ernult, C. Panagopoulos, Nat. Mater. 2017, 16, 898.

10 ] A. K. Yadav, C. T. Nelson, S. L. Hsu, Z. Hong, J. D. Clarkson, C. M. Schlepütz, A. R. Damodaran, P. Shafer, E. Arenholz, L. R. Dedon, D. Chen, A. Vishwanath, A. M. Minor, L. Q. Chen, J. F. Scott, L. W. Martin, R. Ramesh, Nature 2016, 530, 198.

11 ] S. Das, Y. L. Tang, Z. Hong, M. A. P. Gonçalves, M. R. McCarter, C. Klewe, K. X. Nguyen, F. Gómez-Ortiz, P. Shafer, E. Arenholz, V. A. Stoica, S. L. Hsu, B. Wang, C. Ophus, J. F. Liu, C. T. Nelson, S. Saremi, B. Prasad, A. B. Mei, D. G. Schlom, J. Íniguez, P. García-Fernández, D. A. Muller, L. Q. Chen, J. Junquera, L. W. Martin, R. Ramesh, Nature 2019, 568, 368.

12 ] A. Fert, V. Cros, J. Sampaio, Nat. Nanotechnol. 2013, 8, 152.

13 ] J. Sampaio, V. Cros, S. Rohart, A. Thiaville, A. Fert, Nat. Nanotechnol. 2013, 8, 839.

14 ] N. Nagaosa, J. Sinova, S. Onoda, A. H. MacDonald, N. P. Ong, Rev. Mod. Phys. 2010, 82, 1539.

15 ] N. Nagaosa, Y. Tokura, Phys. Scr. 2012, T146, 014020.

16 ] T. H. R. Skyrme, B. F. J. Schonland, Proc. R. Soc. A 1961, 260, 127.

17 ] L. Faddeev, A. J. Niemi, Nature 1997, 387, 58.

18 ] A. Manchon, H. C. Koo, J. Nitta, S. M. Frolov, R. A. Duine, Nat. Mater. 2015, $14,871$.

19 ] A. Soumyanarayanan, N. Reyren, A. Fert, C. Panagopoulos, Nature 2016, 539, 509.

20 ] I. E. Dzyaloshinskii, Sov. Phys. JETP 1957, 5, 1259.

21 ] T. Moriya, Phys. Rev. 1960, 120, 91.

22 ] H. Wilhelm, M. Baenitz, M. Schmidt, U. K. Rößler, A. A. Leonov, A. N. Bogdanov, Phys. Rev. Lett. 2011, 107, 127203.

23 ] X. Z. Yu, N. Kanazawa, Y. Onose, K. Kimoto, W. Z. Zhang, S. Ishiwata, Y. Matsui, Y. Tokura, Nat. Mater. 2011, 10, 106.

24 ] E. Moskvin, S. Grigoriev, V. Dyadkin, H. Eckerlebe, M. Baenitz, M. Schmidt, H. Wilhelm, Phys. Rev. Lett. 2013, 110, 077207.

25 ] C. S. Spencer, J. Gayles, N. A. Porter, S. Sugimoto, Z. Aslam, C. J. Kinane, T. R. Charlton, F. Freimuth, S. Chadov, S. Langridge, J. Sinova, C. Felser, S. Blügel, Y. Mokrousov, C. H. Marrows, Phys. Rev. B 2018, 97, 214406.

26 ] A. N. Bogdanov, U. K. Rößler, Phys. Rev. Lett. 2001, 87, 037203. 
27 ] F. Hellman, A. Hoffmann, Y. Tserkovnyak, G. S. D. Beach, E. E. Fullerton, C. Leighton, A. H. MacDonald, D. C. Ralph, D. A. Arena, H. A. Dürr, P. Fischer, J. Grollier, J. P. Heremans, T. Jungwirth, A. V. Kimel, B. Koopmans, I. N. Krivorotov, S. J. May, A. K. Petford-Long, J. M. Rondinelli, N. Samarth, I. K. Schuller, A. N. Slavin, M. D. Stiles, O. Tchernyshyov, A. Thiaville, B. L. Zink, Rev. Mod. Phys. 2017, 89, 025006.

28 ] S. Woo, K. M. Song, X. Zhang, M. Ezawa, Y. Zhou, X. Liu, M. Weigand, S. Finizio, J. Raabe, M.-C. Park, K.-Y. Lee, J. W. Choi, B.-C. Min, H. C. Koo, J. Chang, Nat. Electron. 2018, 1, 288.

29 ] L. Caretta, M. Mann, F. Büttner, K. Ueda, B. Pfau, C. M. Günther, P. Hessing, A. Churikova, C. Klose, M. Schneider, D. Engel, C. Marcus, D. Bono, K. Bagschik, S. Eisebitt, G. S. D. Beach, Nat. Nanotechnol. 2018, 13, 1154.

30 ] R. Streubel, C. Lambert, N. Kent, P. Ercius, A. T. N'Diaye, C. Ophus, S. Salahuddin, P. Fischer, Adv. Mater. 2018, 30, 1800199.

31 ] I. Bogolubsky, Phys. Lett. A 1988, 126, 511.

32 ] P. Sutcliffe, Rev. Math. Phys. 2018, 30, 1840017.

33 ] M. Hoffmann, B. Zimmermann, G. P. Müller, D. Schürhoff, N. S. Kiselev, C. Melcher, S. Blügel, Nat. Commun. 2017, 8, 308.

34 ] C. Jin, C. Zhang, C. Song, J. Wang, H. Xia, Y. Ma, J. Wang, Y. Wei, J. Wang, Q. Liu, Appl. Phys. Lett. 2019, 114, 192401.

35 ] X. Z. Yu, Y. Tokunaga, Y. Kaneko, W. Z. Zhang, K. Kimoto, Y. Matsui, Y. Taguchi, Y. Tokura, Nat. Commun. 2014, 5, 3198.

36 ] J. C. T. Lee, J. J. Chess, S. A. Montoya, X. Shi, N. Tamura, S. K. Mishra, P. Fischer, B. J. McMorran, S. K. Sinha, E. E. Fullerton, S. D. Kevan, S. Roy, Appl. Phys. Lett. 2016, 109, 022402.

37 ] M. H. Seaberg, B. Holladay, J. C. T. Lee, M. Sikorski, A. H. Reid, S. A. Montoya, G. L. Dakovski, J. D. Koralek, G. Coslovich, S. Moeller, W. F. Schlotter, R. Streubel, S. D. Kevan, P. Fischer, E. E. Fullerton, J. L. Turner, F.-J. Decker, S. K. Sinha, S. Roy, J. J. Turner, Phys. Rev. Lett. 2017, 119, 067403.

38 ] P. Sutcliffe, Phys. Rev. Lett. 2017, 118, 247203.

39 ] X. Zhang, J. Xia, Y. Zhou, X. Liu, H. Zhang, M. Ezawa, Nat. Commun. 2017, 8, 1717.

40 ] D. S. Bouma, Z. Chen, B. Zhang, F. Bruni, M. E. Flatté, A. Ceballos, R. Streubel, L.-W. Wang, R. Q. Wu, F. Hellman, Phys. Rev. B 2020, 101, 014402.

41 ] S. Prakash, C. L. Henley, Phys. Rev. B 1990, 42, 6574.

42 ] K. De'Bell, A. B. MacIsaac, I. N. Booth, J. P. Whitehead, Phys. Rev. B 1997, 55, 15108.

43 ] R. Streubel, N. Kent, S. Dhuey, A. Scholl, S. Kevan, P. Fischer, Nano Lett. 2018, 18, 7428.

44 ] F. Bloch, Z. Physik 1930, 61, 206. Adv. Mater. 2021, 33, 2004830

45 ] R. Wäppling, L. Häggström, Phys. Lett. A 1968, 28, 173.

46 ] V. Senz, R. Röhlsberger, J. Bansmann, O. Leupold, K.-H. Meiwes-Broer, New J. Phys. 2003, 5, 47. 
47 ] K. Maaz, A. Mumtaz, S. K. Hasanain, M. F. Bertino, J. Magn. Magn. Mater. 2010, $322,2199$.

48 ] R. Streubel, D. Makarov, F. Kronast, V. Kravchuk, M. Albrecht, O. G. Schmidt, Phys. Rev. B 2012, 85, 174429.

49 ] I. M. Obaidat, C. Nayek, K. Manna, Appl. Sci. 2017, 7, 1269.

50 ] P. V. Hendriksen, S. Linderoth, P.-A. Lindgard, Phys. Rev. B 1993, 48, 7259.

51 ] B. T. Thole, P. Carra, F. Sette, G. van der Laan, Phys. Rev. Lett. 1992, 68, 1943.

52 ] P. Carra, B. T. Thole, M. Altarelli, X. Wang, Phys. Rev. Lett. 1993, 70, 694.

53 ] R. Streubel, A. T. N’Diaye, K. Srinivasan, A. Ajan, P. Fischer, Appl. Phys. Lett. 2019, $114,162401$.

54 ] R. Streubel, A. T. N'Diaye, K. Srinivasan, A. Kalitsov, S. Jain, A. Ajan, P. Fischer, J. Phys.: Condens. Matter 2021, 33, 104003.

55 ] R. W. Gerchberg, W. O. Saxton, Optik 1972, 35, 227.

56 ] M. R. Teague, J. Opt. Soc. Am. 1983, 73, 1434.

57 ] M. Mansuripur, J. Appl. Phys. 1991, 69, 2455.

58 ] C. Ophus, T. Ewalds, Ultramicroscopy 2012, 113, 88.

59 ] R. Streubel, D. Köhler, R. Schäfer, L. M. Eng, Phys. Rev. B 2013, 87, 054410.

60 ] P. Schoenherr, J. Müller, L. Köhler, A. Rosch, N. Kanazawa, Y. Tokura, M. Garst, D. Meier, Nat. Phys. 2018, 14, 465.

61 ] S. Zhang, G. van der Laan, J. Müller, L. Heinen, M. Garst, A. Bauer, H. Berger, C. Pfleiderer, T. Hesjedal, Proc. Natl. Acad. Sci., U. S. A. 2018, 115, 6386.

62 ] W. Wang, Y. Zhang, G. Xu, L. Peng, B. Ding, Y. Wang, Z. Hou, X. Zhang, X. Li, E. Liu, S. Wang, J. Cai, F. Wang, J. Li, F. Hu, G. Wu, B. Shen, X.-X. Zhang, Adv. Mater. 2016, $28,6887$.

63 ] J. C. Loudon, A. C. Twitchett-Harrison, D. Cortés-Ortuno, M. T. Birch, L. A. Turnbull, A. Štefančič, F. Y. Ogrin, E. O. Burgos- Parra, N. Bukin, A. Laurenson, H. Popescu, M. Beg, O. Hovorka, H. Fangohr, P. A. Midgley, G. Balakrishnan, P. D. Hatton, Adv. Mater. 2019, 31, 1806598.

64 ] Y. Yao, B. Ding, J. Cui, X. Shen, Y. Wang, W. Wang, R. Yu, Appl. Phys. Lett. 2019, 114, 102404.

65 ] A. Leonov, T. Monchesky, J. Loudon, A. Bogdanov, J. Phys. Cond. Matter 2016, 28, 35LT01. 